\title{
STIFF SYSTEMS OF ORDINARY DIFFERENTIAL EQUATIONS. III. PARTIALLY STIFF SYSTEMS
}

\author{
J. J. MAHONY AND J. J. SHEPHERD
}

(Received 8 November 1979)

(Revised 2 March 1981)

\begin{abstract}
The partially stiff system of ordinary differential equations

$$
\begin{aligned}
e \dot{x} & =A x+B y+r_{1}, \\
\dot{y} & =C x+D y+r_{2}
\end{aligned}
$$

is studied by the methods developed in the earlier papers in this series. Here $e$ is a small positive parameter, $\mathrm{x}$ and $\mathrm{y}$ are $n$ - and $\boldsymbol{m}$-vectors respectively, and $\boldsymbol{A}$ is nonsingular. $\mathrm{A}$ useful basis for the solution space of the homogeneous system is constructed and the method of variation of parameters is used to obtain useful representations of all solutions. Sufficient conditions are derived under which the formal approximation

$$
\begin{aligned}
& 0=A \mathrm{x}+B \mathrm{y}+\mathrm{r}_{1}, \\
& \dot{\mathrm{y}}=C \mathrm{x}+D \mathrm{y}+\mathrm{r}_{2}
\end{aligned}
$$

is close to the actual solution. It is found that purely imaginary eigenvalues for $A$ require more stringent requirements for the formal technique to be valid. A brief discussion of the case when $A$ is singular shows that there are a great number of possibilities requiring consideration for a general theory. It is suggested that local computation of such cases is likely to be the most effective weapon for any specific system.
\end{abstract}

\section{Introduction}

In a previous paper [2], referred to below as Part I, we have considered the question of constructing useful forms of a basis for the solution space of the completely stiff linear system

$$
\varepsilon \dot{\mathbf{x}}=A \mathbf{x}
$$

C. Copyright Australian Mathematical Society 1982 
Here $A$ is a continuously differentiable $n \times n$ matrix for a set of positive values of $\varepsilon$ in a neighbourhood of zero, possessing $n$ differentiable eigenvalue functions $\lambda_{1}, \ldots, \lambda_{n}$, not necessarily distinct, and a corresponding continuously differentiable eigenvector basis $S=\left(s_{1}, \ldots, s_{n}\right)$ such that, on the interval of $t$-values under consideration, $A$ has the canonical form

$$
A=S \Lambda S^{-1}
$$

where $\Lambda=\operatorname{diag}\left(\lambda_{1}, \ldots, \lambda_{n}\right)$.

The limitations of this particular set of assumptions have been discussed at some length in the reference given above. On any interval for which an invariant ordering of the real parts of the distinct $\lambda_{i}$ holds, we have shown that there is a fundamental matrix for (1.1) of the form

$$
\{S+o(1)\} Z \text {, }
$$

where

$$
Z=\operatorname{diag}\left\{\exp \left(\varepsilon^{-1} \int^{t} \lambda_{i} d s\right)\right\} \stackrel{\text { defn }}{=} \operatorname{diag}\left\{\exp \zeta_{i}\right\}
$$

where the lower limit of integration is chosen arbitrarily. In the above, $S$ consists of appropriately chosen eigenvector functions of $A$.

In general, there is no such simple basis on intervals where the partial ordering of eigenvalue functions is not maintained. However, an algebraic algorithm for connecting the fundamental matrices of the form (1.3) on abutting intervals was devised, and the resulting structure of the uniform basis obtained was displayed.

A later paper [3], referred to as Part II, used the basis (1.3) to consider the circumstances under which boundary value problems for the inhomogeneous system

$$
\varepsilon \dot{\mathbf{x}}=A \mathbf{x}+\mathbf{r}
$$

would have solutions, and to obtain qualitative information about the properties of such solutions.

These investigations were motivated by a desire to provide a well-founded theoretical basis for possible numerical packages applicable to the solution of large order stiff systems of nonlinear ordinary differential equations. Our viewpoint has been to suppose that a given numerical package has provided a candidate approximate solution (in some approximate sense) and then to enquire as to when we can be sure that the exact problem has a neighbouring solution.

Such questions are usually answered in the affirmative by the process of linearizing the problem about the candidate approximate solution and using an iterative procedure such as the Contraction Mapping Theorem. However, in [3] we demonstrated that there do exist problems where the candidate approximate 
solution may be a good approximation to a solution of the problem, but that more delicate fixed-point theorems may be needed to establish this property.

The assumptions involved in this previous work, that the system under consideration is completely stiff in the sense of (1.1), are far too restrictive, in that many systems do not display this property. In this paper we will use the methods developed so far to extend our results to deal with the partially stiff homogeneous system

$$
\begin{aligned}
\varepsilon \dot{\mathbf{x}} & =A \mathbf{x}+B \mathbf{y}, \\
\dot{\mathbf{y}} & =C \mathbf{x}+D \mathbf{y},
\end{aligned}
$$

where $\mathbf{x}$ and $\mathbf{y}$ are $n$ - and $m$-vectors respectively, and $A, B, C$ and $D$ are $n \times n$, $n \times m, m \times n$ and $m \times m$ matrices respectively, defined on an interval of $t$-values $J$, for each $\varepsilon$ in some neighbourhood of zero, and having continuity and differentiability properties to be specified. We will establish the existence of a basis for the solution space of (1.6) and (1.7) in a form that is suitable for the description of the qualitative behaviour of solutions of this system. Questions of obtaining suitable particular integrals of the inhomogeneous counterpart of this, and of satisfying given boundary conditions, then reduce to problems very similar to those considered for the completely stiff system, and we merely indicate how such problems may be tackled. We are able to give a sufficient set of conditions to ensure that formal asymptotic methods give useful approximations.

For the partially stiff system (1.6) and (1.7), one might expect that there would also be similar rapidly varying solutions to those of (1.1), because of the presence of the terms $\varepsilon \dot{\mathbf{x}}=A \mathbf{x}$. Moreover, if the $\mathbf{x}$-component of the solution exhibits such behaviour, we might reasonably expect a similar behaviour in the $y$-component. This becomes clear when we observe that if $\Psi$ is a fundamental solution of $\dot{\Psi}=D \Psi$, then

$$
\mathbf{y}=\Psi \int^{t} \Psi^{-1} C \mathbf{x}
$$

satisfies (1.7) for any suitable $\mathbf{x}$. If $\mathbf{x}$ exhibits the rapidly varying behaviour alluded to above, and if the terminals of integration in (1.8) can be chosen appropriately, the integral may be dominated by the properties of the integrand at $t$.

This suggests that we can generate stiff solutions, that is, solutions whose general behaviour is that of a function whose derivative is large in comparison with the value of the function itself, by considering solutions of

$$
\varepsilon \dot{\mathbf{x}}=A \mathbf{x}+B \Psi \int^{t} \Psi^{-1} C \mathbf{x} .
$$


We show in Section 2 that it is possible to organize the integral in (1.8) in such a way that the expectations that there are $n$ linearly independent such solutions are confirmed, at least under assumptions comparable to those of Part $I$, with the further important restriction that $A$ should not be singular. The construction in Section 2 is restricted to intervals on which the real parts of all eigenvalue functions are one-signed but the results are extended, with some restriction, in Section 4.

We also might expect the existence of solutions that do not exhibit such rapidly changing behaviour. The results obtained in Part II indicate that the solutions of (1.7) with this property look like the solutions of

$$
\mathbf{x}=-A^{-1} B \mathbf{y}
$$

for most $t$-values, so that one might look for solutions resembling those of

$$
\dot{\mathbf{y}}=\left(D-C A^{-1} B\right) \mathbf{y}
$$

We show that this expectation too is confirmed, by applying the methods that have proved effective in Parts I and II.

However, there is a major difference in the present case, which limits the generality of the results obtained. If any of the eigenvalues of $A$ is purely imaginary for the range of $t$-values under consideration (and an appropriate range of $\varepsilon$ values), the results are establishable only for compact intervals of integration. The problem is not merely one of the method of approach, for there are known examples where cumulative small errors arising in formal methods become significant. Thus this failure of the techniques that have been applied in this series of papers indicates that very delicate questions are involved, and each example must be considered on its individual merits.

We close this introduction with a comment regarding the notation to be adopted. In Parts I and II, we took pains to show that the results obtained are useful in a context in which the data was defined for a limited set of values of $\varepsilon$, a situation occurring frequently in numerical applications. This was done at the expense of a good deal of complicated use of language and symbolism. In this paper, we will revert to the simpler language used more commonly in the asymptotic analysis of singular perturbation problems, in order that the presentation might be clearer. However, it must be borne in mind that, under the conditions described above, the results obtained could be restated in the form adopted in the earlier papers. In particular, we do not need our assumptions to hold for all $\varepsilon$ in a neighbourhood of zero, but merely for some (possibly as few as one) value of $\varepsilon$ in this neighbourhood. 


\section{The stiff partial basis}

We begin our construction of a basis for the solution space of (1.6) and (1.7) by constructing a partial basis consisting principally of stiff solutions, as described in Section 1. We will term this partial basis, for convenience, the stiff partial basis, and expect that its behaviour will be largely dominated by the solution of (1.1).

If we let $\Psi$ be the fundamental matrix as defined in (1.8), we see that (1.6) and (1.7) may be satisfied automatically by any solution $\mathrm{x}$ given by

$$
\varepsilon \dot{\mathbf{x}}=A \mathbf{x}+B \Psi \int^{t} \Psi^{-1} C \mathbf{x}
$$

with the corresponding $y$ component being given by (1.8).

Because $A$ will play such a central role in our construction, we briefly state our assumptions regarding this matrix, on the interval $\bar{J}$ which we choose, for definiteness, to be $\left[t_{0}, t_{1}\right]$.

AssumPTION 1. There exists $\varepsilon \in\left(0, \varepsilon_{0}\right]$ such that $A(t, \varepsilon)$ has $n$ linearly independent eigenvectors $\mathrm{s}_{i}(t, \varepsilon)$ and corresponding eigenvalues $\lambda_{i}(t, \varepsilon)$, not necessarily distinct, with both sets of functions being continuously differentiable on $\bar{J}$.

This assumption gives us the canonical form (1.2) on $\bar{J}$ which will prove to be of considerable use.

We now seek solutions of the integrodifferential equation (2.1) that may be expressed in the form

$$
\mathbf{x}=S M Z \mathbf{b},
$$

where $S$ are the eigenvectors of $A, Z$ is given by (1.4) with

$$
\zeta_{i}=\frac{1}{\varepsilon} \int_{t_{0}}^{t} \lambda_{i}(s, \varepsilon) d s
$$

while $M$ is an $n \times n$ matrix to be specified and $\mathbf{b}$ is an $n$-vector function to be determined. Direct substitution of (2.2) into (2.1) gives

$$
\begin{aligned}
\dot{\mathbf{b}}= & Z^{-1} M^{-1}\left[\varepsilon^{-1}(\Lambda M-M \Lambda)-\dot{M}-S^{-1} \dot{S} M\right] Z \mathbf{b} \\
& +\varepsilon^{-1} Z^{-1} M^{-1} S^{-1} B \Psi \int^{t} \Psi^{-1} C S M Z \mathbf{b},
\end{aligned}
$$

and thus, on integration, we obtain

$$
\begin{aligned}
\mathbf{b}= & \mathbf{e}_{i}+\int^{t} Z^{-1} M^{-1}\left[\varepsilon^{-1}(\Lambda M-M \Lambda)-\dot{M}-S^{-1} \dot{S} M\right] Z \mathbf{b} \\
& +\varepsilon^{-1} \int^{t} Z^{-1} M^{-1} S^{-1} B \Psi \int^{s} \Psi^{-1} C S M Z \mathbf{b},
\end{aligned}
$$


where the lower terminals of integration are yet to be specified, while $\mathbf{e}_{i}$, $i=1,2, \ldots, n$ is the usual basis in $R^{n}$. By choosing $M$ to have an appropriate block-diagonal form (see Part I, Section 3) we may ensure that $\Lambda M=M \lambda$ for given $\varepsilon \in\left(0, \varepsilon_{0}\right]$ and each $t \in \bar{J}$.

Following the procedure of Part I, we make the further substitution

$$
\boldsymbol{\beta}=\exp \left(-\zeta_{i}\right) Z \mathbf{b}
$$

for each given $i$, so that, with the above choice of $M$, we may write (2.4) as

$$
\begin{aligned}
\boldsymbol{\beta}= & \mathbf{e}_{i}-\exp \left(-\zeta_{i}\right) Z \int^{t} Z^{-1} M^{-1}\left[\dot{M}+S^{-1} \dot{S} M\right] \exp \left(\zeta_{i}\right) \boldsymbol{\beta} \\
& +\varepsilon^{-1} \exp \left(-\zeta_{i}\right) Z \int^{t} Z^{-1} M^{-1} S^{-1} B \Psi \int^{s} \Psi^{-1} C S M \exp \left(\zeta_{i}\right) \boldsymbol{\beta} .
\end{aligned}
$$

If we denote by the symbol $I_{j}$ a unit matrix that selects the $j$ th row of any matrix $P$ by premultiplication $I_{j} P$, we may write the $j$ th component of the last term of (2.7) in the form

$$
Q_{j} \beta=\varepsilon^{-1} \exp \left(\zeta_{j}-\zeta_{i}\right) \int^{t} \exp \left(-\zeta_{j}\right) I_{j} M^{-1} S^{-1} B \Psi \int^{s} \Psi^{-1} C S M \exp \left(\zeta_{i}\right) \boldsymbol{\beta} .
$$

Evidently $Q$, represents a linear map on the Banach space $B_{e}$ of continuous vector functions $\beta(t, \varepsilon)$ normed, for each $\varepsilon>0$, by

$$
\|\beta\|=\max _{i}\left\{\sup _{t}\left|\beta_{i}(t, \varepsilon)\right|\right\},
$$

and it is clear that the last term of (2.7) may be written as the direct sum

$$
\bigoplus_{j} Q_{j} \beta \text {. }
$$

To investigate further the properties of these component maps, as well as those of the second term of (2.7), we need to specify the lower terminals of integration involved in these. Such a choice may be made under appropriate assumptions about the properties of the eigenvalues of $A$ on $\bar{J}$, given as below.

AsSUMPTION 2. There exists an $\varepsilon \in\left(0, \varepsilon_{0}\right]$ such that, for all $t \in \bar{J}$, and all eigenvalue functions $\lambda_{i}$ and $\lambda_{j}$ of $A$, the zeros of $\operatorname{Re} \lambda_{i}, \operatorname{Im} \lambda_{i}, \operatorname{Re}\left(\lambda_{i}-\lambda_{j}\right)$ and $\operatorname{Im}\left(\lambda_{i}-\lambda_{j}\right)$ on $\bar{J}$ are finite in number and of finite order, while

(i) either $\operatorname{Re} \lambda_{i}>0$ or $\operatorname{Re} \lambda_{i}<0$ or $\operatorname{Re} \lambda_{i} \equiv 0$ on $\bar{J}$,

(ii) either $\operatorname{Re}\left(\lambda_{i}-\lambda_{j}\right) \geqslant 0$ or $\operatorname{Re}\left(\lambda_{i}-\lambda_{j}\right)<0$ or $\operatorname{Re}\left(\lambda_{i}-\lambda_{j}\right) \equiv 0$ on $\bar{J}$, and

(iii) $\lambda_{i} \neq 0$ anywhere on $\bar{J}$.

These will suffice for the construction of our basis on $\bar{J}$. The second has already been employed in Part I for the construction of the basis to the solution space of the completely stiff system (1.1). It should also be noted that (iii) really refers only to real eigenvalues; for the vanishing of a complex eigenvalue would violate our assumption regarding the $s_{i}$ spanning $R^{n}$ for all $t \in \bar{J}$. 
With these assumptions, we may define the lower limit of integration in (2.1), and hence in the inner integral of $(2.8)$ to be $\tau_{i}$, where

$$
\tau_{i}=\left\{\begin{array}{l}
t_{0} \text { when } \operatorname{Re} \lambda_{i} \geqslant 0 \text { or } \operatorname{Re} \lambda_{i} \equiv 0, \\
t_{1} \text { when } \operatorname{Re} \lambda_{i} \leqslant 0 .
\end{array}\right.
$$

For the second term of (2.7), and hence the outer integral of (2.8), we choose the lower terminal to be $\sigma_{i j}$, where

$$
\sigma_{i j}=\left\{\begin{array}{l}
t_{0} \text { when } \operatorname{Re}\left(\lambda_{i}-\lambda_{j}\right) \geqslant \text { or } \operatorname{Re}\left(\lambda_{i}-\lambda\right) \equiv 0, \\
t_{1} \text { when } \operatorname{Re}\left(\lambda_{i}-\lambda_{j}\right)<0 .
\end{array}\right.
$$

These choices ensure that, in general, the integrals involved are dominated by the values of the integrand at $t$ in the sense that neighbourhoods of other points in the range of integration do not contribute to significantly large order. Moreover, they show that there occur up to four types of iterated integral in the maps $Q_{j}$, depending on the properties of the eigenvalues concerned. Because of the complexity of the analysis involved, we investigate the properties of these maps below for two choices of $\tau_{i}$ and $\sigma_{i j}$; the others follow by analogy.

When $\tau_{i}=t_{0}$ and $\sigma_{i j}=t_{0}$, we have

$$
\begin{aligned}
Q_{j} \beta & =\varepsilon^{-1} \exp \left(\zeta_{j}-\zeta_{i}\right) \int_{t_{0}}^{t} \exp \left(-\zeta_{j}\right) I_{j} M^{-1} S^{-1} B \Psi \int_{t_{0}}^{s} \Psi^{-1} C S M \exp \left(\zeta_{i}\right) \beta d u d s \\
& =\varepsilon^{-1} \exp \left(\zeta_{j}-\zeta_{i}\right) \int_{u=t_{0}}^{u=t}\left\{\int_{s=u}^{s=t} \exp \left(-\zeta_{j}\right) I_{j} M^{-1} S^{-1} B \Psi d s\right\} \Psi^{-1} C S M \exp \left(\zeta_{i}\right) \beta d u
\end{aligned}
$$

on a change of the order of integration. Integration-by-parts applied to the inner integral above now gives

$$
\begin{aligned}
Q_{j} \beta= & \exp \left(\zeta_{j}-\zeta_{i}\right) \int_{u=t_{0}}^{u=t}\left[\lambda_{i j}^{-1} \exp \left(-\zeta_{j}\right) I_{j} M^{-1} S^{-1} B \Psi\right]_{u}^{t} \Psi^{-1} C S M \exp \left(\zeta_{i}\right) \beta d u \\
& +\exp \left(\zeta_{j}-\zeta_{i}\right) \int_{u=t_{0}}^{u=t}\left\{\int_{s=u}^{s=t} \exp \left(-\zeta_{j}\right) \frac{d}{d s}\left(\lambda_{j}^{-1} I_{j} M^{-1} S^{-1} B \Psi\right) d s\right\} \\
& \times \Psi^{-1} C S M \exp \left(\zeta_{i}\right) \beta d u
\end{aligned}
$$

It is now a straightforward matter to invoke results given in Erdélyi [1] and the Riemann-Lebesgue lemma to show that, provided $\Psi^{-1} C S M$ and $d\left(\lambda_{j}^{-1} I_{j} M^{-1} S^{-1} B \Psi\right) / d s$ are respectively bounded and absolutely integrable on $\bar{J}$, with values independent of $\varepsilon$, the second term of (2.15) is bounded above by a term

$$
o(1)\|\boldsymbol{\beta}\|
$$

for all $\lambda_{i}$ and $\lambda_{j}$ giving the above choices of $\tau_{i}$ and $\sigma_{i j}$. Here, $o(1)$ is a term that vanishes with $\varepsilon$ uniformly with repect to $t \in \bar{J}$. 
Explicit evaluation of the first term of (2.15) gives

$$
\begin{aligned}
& -\exp \left(-\zeta_{i}\right) \lambda_{j}^{-1} I_{j} M^{-1} S^{-1} B \Psi \int_{t_{0}}^{t} \Psi^{-1} C S M \exp \left(\zeta_{i}\right) \boldsymbol{\beta} d u \\
& +\exp \left(\zeta_{j}-\zeta_{i}\right) \int_{t_{0}}^{t} \lambda_{j}^{-1} \exp \left(\zeta_{i}-\zeta_{j}\right) I_{j} M^{-1} S^{-1} B C S M \beta d u
\end{aligned}
$$

The second of these may be incorporated into the second term of (2.7) (or, more accurately, the $j$ th component of this term) while, when $\operatorname{Re} \lambda_{i} \neq 0$ on $J$, the first term is bounded by a term of the form of (2.16) under appropriate assumptions about the integrand. When $\operatorname{Re} \lambda_{i} \equiv 0$, however, this term is bounded only, in terms of the norm $\|\cdot\|$.

When $\tau_{i}=t_{0}$, but $\sigma_{i j}=t_{1}$, we may write, after interchange of the order of integration,

$$
\begin{aligned}
Q_{j} \beta= & -\varepsilon^{-1} \exp \left(\zeta_{i}-\zeta_{j}\right) \int_{t_{0}}^{t}\left\{\int_{t}^{t_{1}} \exp \left(-\zeta_{j}\right) I_{j} M^{-1} S^{-1} B \Psi d s\right\} \Psi^{-1} C S M \exp \left(\zeta_{i}\right) \beta d u \\
& -\varepsilon^{-1} \exp \left(\zeta_{j}-\zeta_{i}\right) \int_{t}^{t_{1}}\left\{\int_{u}^{t_{1}} \exp \left(-\zeta_{j}\right) I_{j} M^{-1} S^{-1} B \Psi d s\right\} \Psi^{-1} C S M \exp \left(\zeta_{i}\right) \beta d u
\end{aligned}
$$

Upon integrating by parts, as applied above, and estimating the integrals produced, we may show that, in this instance,

$$
\begin{aligned}
Q_{j} \beta= & -\exp \left(-\zeta_{i}\right) \lambda_{j}^{-1}\left(I_{j} M^{-1} S^{-1} B \Psi\right) \int_{t_{0}}^{t} \Psi^{-1} C S M \exp \left(\zeta_{i}\right) \beta d u \\
& -\exp \left(\zeta_{j}-\zeta_{i}\right) \int_{t}^{t_{1}} \lambda_{j}^{-1} \exp \left(\zeta_{i}-\zeta_{j}\right) I_{j} M^{-1} S^{-1} B C S M \beta d u+o(1)\|\beta\| .
\end{aligned}
$$

Analogous results hold for the other two choices of $\tau_{i}$ and $\sigma_{i j}$ and, as we have said, we leave the details to the reader.

The results obtained above imply that (2.7) may be written as

$$
\boldsymbol{\beta}=\mathbf{e}_{i}+\mathfrak{T} \boldsymbol{\beta}
$$

where the map $\mathscr{N}: \mathscr{B}_{\varepsilon} \rightarrow \mathscr{B}_{\varepsilon}$ is given by

$$
\mathfrak{K} \boldsymbol{\beta}=\mathfrak{L} \boldsymbol{\beta}-\mathfrak{K} \boldsymbol{\beta}+\boldsymbol{T} \boldsymbol{\beta},
$$

where

$$
\|T \boldsymbol{\beta}\|=o(1)\|\beta\| \text { for all } \boldsymbol{\beta} \in \mathscr{B}_{e},
$$

while $\mathcal{K}$ and $\mathcal{L}$ are defined by

$$
\mathscr{K} \beta=\underset{j}{\bigoplus} \mathfrak{K}_{j} \beta \text { and } \mathcal{L} \beta=\bigoplus_{j} \mathcal{L}_{j} \beta
$$


with

$$
\mathcal{K}_{j} \beta=\exp \left(\zeta_{j}-\zeta_{i}\right) \int_{\sigma_{i}}^{t} \exp \left(\zeta_{i}-\zeta_{j}\right) I_{j} M^{-1}\left[\dot{M}+\left(S^{-1} \dot{S}+\Lambda^{-1} S^{-1} B C S\right) M\right] \beta d u
$$

and

$$
\varrho_{j} \beta=-\exp \left(-\zeta_{i}\right) \lambda_{i}^{-1} I_{j} M^{-1} S^{-1} B \Psi \int_{\sigma_{i j}}^{t} \Psi^{-1} C S M \exp \left(\zeta_{i}\right) \beta d u
$$

respectively.

If we parallel the procedure of Part $\mathrm{I}$, and choose $M$ to satisfy

$$
\dot{M}_{\alpha}+\left(S^{-1} \dot{S}+S^{-1} A^{-1} B C S\right)_{\alpha} M_{\alpha}=0,
$$

where subscripts $\alpha$ denote the restriction of quantities to the subspace spanned by eigenvectors corresponding to eigenvalues that are all equal, together with a nonsingular initial choice of $M$, we may ensure, by means of Assumption 2, that $\mathcal{H}_{j}^{2} \equiv 0$, while $\mathcal{H}_{j} \mathcal{H}_{k}$ are contractive on $\mathscr{B}_{\varepsilon}$ with contraction parameter $o(1)$ in the sense of (2.16). Thus $\mathcal{H}^{2}$ is contractive on $\mathscr{B}_{\varepsilon}$.

There remains the linear map $\mathcal{L}$ given by $(2.25)$. When $\operatorname{Re} \lambda_{i} \neq 0$, this too is contractive on $\mathscr{G}_{e}$, by the choice of $\sigma_{i j}$, while it is only bounded when $\operatorname{Re} \lambda_{i} \equiv 0$ on $\bar{J}$.

Combining these results, we may write the equation (2.20) as

$$
\boldsymbol{\beta}=\mathbf{e}_{i}+\mathfrak{T} \mathbf{e}_{i}+\mathscr{Q} \beta+\mathscr{T} \boldsymbol{\beta}
$$

where $\mathcal{T}$ is contractive on $\mathscr{B}_{e}$ in the sense of (2.16), while $\mathcal{Q}$ has an integral representation

$$
\mathcal{Q} \beta=U \int_{t_{0}}^{t} V \beta d u
$$

for matrices $U$ and $V$ depending on the matrices used above. When $\mathbb{Q}$ has no pure imaginary eigenvalues, $\mathcal{Q} \equiv 0$, while it is clear from (2.21) that

$$
\mathcal{Q} \mathbf{e}_{i}=o(1)
$$

uniformly for $t \in \bar{J}$.

It is clear from (2.28) that, under suitable boundedness assumptions about $U$ and $V$,

$$
\left\|\mathbb{Q}^{N} \boldsymbol{\beta}\right\| \leqslant\left(t_{1}-t_{0}\right)^{N}(N !)^{-1}\|\beta\|,
$$

so that, for $N$ large enough and $\left|t_{1}-t_{0}\right|$ bounded, we may iterate (2.27) to a point where the right hand side is a contraction on $\mathscr{B}_{e}$ (with contraction parameter dependent on $N$ ). This yields the existence of a unique fixed point to this right-hand side, and hence a unique solution to (2.27). However, this does not give us the estimates we desire for $\boldsymbol{\beta}-\mathbf{e}_{i}$, so we proceed as follows. 
On any interval $\left[t_{0}, t_{0}+\Delta\right]$ we may write, for the solution $\beta$ of (2.27) already constructed, with norms $\|\cdot\|^{*}$ defined on this interval,

$$
\left\|\boldsymbol{\beta}-\mathbf{e}_{i}\right\|^{*} \leqslant o(1)+(o(1)+\Delta)\left\|\boldsymbol{\beta}-\mathbf{e}_{i}\right\|^{*},
$$

where the $o(1)$ are terms that vanish as $\varepsilon \rightarrow 0$. This yields

$$
\left\|\boldsymbol{\beta}-\mathbf{e}_{i}\right\|^{*}=o(1)
$$

for any finite $\Delta<1 / 2$, which gives the desired estimate on $\left[t_{0}, t_{0}+\Delta\right]$. The same procedure may now be applied to $\left[t_{0}+\Delta, t_{0}+2 \Delta\right]$, and thence on to $t_{1}$, provided $t_{1}-t_{0}$ is bounded independent of $\varepsilon$.

The results obtained so far may now be applied to yield our existence result for the stiff partial basis for the system (1.6) and (1.7).

THEOREM 1. Let Assumptions 1 and 2 hold, and let $\dot{B}$ and $B, C$ be absolutely integrable and bounded respectively, independently of $\varepsilon$ on $\bar{J}$. If any $\lambda_{i}$ has its real part identically zero, let $\bar{J}$ be bounded independent of $\varepsilon$.

Then there exist $n$ solutions $x_{r}$ and $y_{r}$ of the system (1.6) and (1.7) which are linearly independent on $\bar{J}$ for appropriate $\varepsilon \in\left(0, \varepsilon_{0}\right]$, and which are given by

$$
\begin{gathered}
\mathbf{x}_{r}=S M \exp \left(\zeta_{r}\right)\left[\mathbf{e}_{r}+o(1)\right], \\
\mathbf{y}_{r}=\Psi \int_{\tau_{r}}^{t} \Psi^{-1} C S M \exp \left(\zeta_{r}\right)\left[\mathbf{e}_{r}+o(1)\right],
\end{gathered}
$$

$r=1,2, \ldots, n$, where $\tau_{r}$ is defined by (2.11), $\Psi$ is as in (2.1), and the o(1) terms vanish with $\mathrm{E}$ uniformly for $t \in \bar{J}$.

Proof. Our existence result for the equation (2.20), together with the estimate (2.32) over all of $\bar{J}$, give the result (2.33), when we note (2.2) and (2.6). The second (2.34) follows directly from (2.1). The linear independence of the set is inherited from that of the $\mathbf{x}_{r}$.

\section{REMARKS.}

1. It is important to note that in the above, we need the Assumption 2(iii). This in fact ensures that $A^{-1}$ exists on $\bar{J}$; we will examine the consequences of relaxing this condition later.

2. The hypotheses of the theorem make no mention of the properties of $M, \Psi$ and $\Lambda$. That such properties do hold is ensured by the definition and construction of these quantities and is inherited from this.

3. Note that the varied equation for $M$ given by (2.26) means that we have to select different multiples of the eigenvectors $s_{i}$ of $A$ for this partially stiff system from that choice adopted for the completely stiff system (1.1). The choice (2.26), which may be written

$$
\dot{M}_{\alpha}+\left(S^{-1} \dot{S}+S^{-1} A^{-1} B C S\right)_{\alpha} M_{\alpha}=0,
$$


gives us, on each eigenvalue subspace, the appropriate generalization of the Liouville result.

4. It is important to note from (2.34) that, under the hypotheses above, the $\mathbf{y}_{r}$ behave somewhat like $o(1) \exp \left(\zeta_{r}\right)$, that is, they display the stiff behaviour of the $\mathbf{x}_{r}$, though at a higher order in small terms.

\section{The nonstiff partial basis}

We continue our construction of the basis for the solution space of (1.6) and (1.7) by considering solutions that we will term nonstiff, and hence a nonstiff partial basis for this solution space, in the sense that they are largely dominated by the solution of $A \mathbf{x}+B \mathbf{y}=\mathbf{0}$ and $\dot{\mathbf{y}}=C \mathbf{x}+D \mathbf{y}$.

Given that Assumptions 1 and 2 hold, we may, by exploiting the results of Part I, write down a particular integral of (1.6) in the form

$$
\mathbf{x}=\varepsilon^{-1} X Z \int^{t} Z^{-1} X^{-1} B \mathbf{y},
$$

where

$$
X=S(I+o(1))
$$

uniformly on $\bar{J}$, while $Z$ is given by (1.4) for $\zeta_{i}$ given by (2.3). Then (1.7) becomes

$$
\dot{\mathbf{y}}=D \mathbf{y}+\varepsilon^{-1} C X Z \int^{t} Z^{-1} X^{-1} B \mathbf{y}
$$

which may be converted to

$$
\mathbf{y}=\chi \mathbf{e}_{i}+\chi \int^{t} \chi^{-1} C\left[A^{-1} B y+\varepsilon^{-1} X Z \int^{s} Z^{-1} X^{-1} B y\right],
$$

where $\chi$ is a fundamental matrix satisfying the equation

$$
\dot{\chi}=\left(D-C A^{-1} B\right) x,
$$

and $\mathrm{e}_{i}, i=n+1, \ldots, n+m$, form the usual basis vectors in $R^{m}$. As yet, we have not specified the lower terminal of integration in (3.4).

For each $\varepsilon>0$, the right-hand side of (3.4) represents a bounded linear map on $\mathscr{B}_{e}$ in terms of the norm (2.9). To apply the contraction mapping principle to this, however, we require improved estimates of this operator norm and, to this end, we consider the properties of the double integral in (3.4), namely

$$
M \mathbf{y}=\varepsilon^{-1} \chi \int^{t} \chi^{-1} C X Z \int^{s} Z^{-1} X^{-1} B \mathbf{y},
$$


which is also a bounded linear map on $\mathscr{B}_{\varepsilon}$. Recalling the procedure adopted in Section 2, we see that this map may be written as the direct sum of maps $\boldsymbol{M}_{j}$, where

$$
M_{j} \mathbf{y}=\varepsilon^{-1} \chi \int^{t} \chi^{-1} C X I_{j} \exp \left(\zeta_{j}\right) \int^{s} \exp \left(-\zeta_{j}\right) X^{-1} B \mathbf{y}
$$

and $I_{j}$ is the identity matrix as defined in Section 2 . We may now specify the terminals involved in the integrals of (3.7). We choose the lower terminal in each case to be $\mu_{j}$, where

$$
\mu_{j}=\left\{\begin{array}{l}
t_{0} \text { if } \operatorname{Re} \lambda_{j} \leqslant 0 \text { or } \operatorname{Re} \lambda_{j} \equiv 0 \text { on } \bar{J}, \\
t_{1} \text { if } \operatorname{Re} \lambda_{j}>0 \text { on } \bar{J} .
\end{array}\right.
$$

By reversing the order of integration, and integrating by parts in (3.7), we obtain, for $\mu_{j}=t_{0}$,

$$
\begin{aligned}
M_{j} \mathrm{y}= & \chi \int_{t_{0}}^{t}\left[\chi^{-1} C X \lambda_{j}^{-1} I_{j} \exp \left(\zeta_{j}\right)\right]_{u}^{t} \exp \left(-\zeta_{j}\right) X^{-1} B \mathbf{y} \\
& -\chi \int_{t_{0}}^{t} \int_{u}^{t} \frac{d}{d s}\left\{\chi^{-1} C X \lambda_{j}^{-1} I_{j} e^{\zeta_{j}}\right\} \exp \left(-\zeta_{j}\right) X^{-1} B \mathbf{y},
\end{aligned}
$$

where, by Assumption $2, \lambda_{j} \neq 0$ on $\bar{J}$.

When $\operatorname{Re} \lambda_{j} \neq 0$, we may, by a second reversal of integration, show that the second term in (3.9) is bounded in modulus by a term of the form

$$
o(1)\|\mathbf{y}\| \text {, }
$$

where the $o(1)$ vanishes as $\varepsilon \rightarrow 0$ uniformly for $t \in \bar{J}$. When $\operatorname{Re} \lambda_{j} \equiv 0$, a similar result holds by applying the Riemann-Lebesgue lemma to the inner integral.

The first term may be evaluated explicitly to give

$$
M_{j} \mathbf{y}=C X I_{j} \lambda_{j}^{-1} \exp \left(\zeta_{j}\right) \int_{t_{0}}^{t} \exp \left(-\zeta_{j}\right) X^{-1} B \mathbf{y}-\chi \int_{t_{0}}^{t} \chi^{-1} C I_{j} A^{-1} B \mathbf{y}+o(1)\|\mathbf{y}\|,
$$

where we have noted the canonical form (1.2), and the result (3.2). The last term represents a linear map on $\mathscr{B}_{e}$ bounded in modulus by a term of the form of (3.10).

It is not a difficult matter to show that analogous results hold for $\mu_{j}=t_{1}$. We thus obtain, on incorporating these results into the equation (3.6), that

$$
\mathbf{y}=\chi \mathbf{e}_{i}+\mathcal{C} \mathbf{y}+T \mathbf{y}
$$

where $T$ is contractive on $\mathscr{B}_{\varepsilon}$ in the sense of (3.10), while $\mathcal{C}$ is a linear map that is non-zero only when $A$ has purely imaginary eigenvalues, and is given for such eigenvalues $\lambda_{j}$ by $\mathcal{C y}=\oplus, \mathcal{C}_{j} \mathbf{y}$ where

$$
\mathcal{C}_{j} \mathbf{y}=C X I, \lambda_{j}^{-1} \exp \left(\zeta_{j}\right) \int_{t_{0}}^{t} \exp \left(-\zeta_{j}\right) X^{-1} B \mathbf{y}
$$


We may now apply a similar construction procedure to (3.12) to that used in Section 2, to obtain the existence of a unique fixed point $y \in \mathscr{B}_{\varepsilon}$. Moreover, since $\mathcal{C}_{j}$ has the particular form (3.13), the estimation procedure used there may also be applied here, to give the result that

$$
y=\chi \mathbf{e}_{i}+o(1)
$$

We thus arrive at our existence result for the non-stiff partial basis for the solution space of (1.0) andu (1.7).

TheOREM 2. Let Assumptions 1 and 2 hold, with $\dot{C}$ and $B$ absolutely integrable and bounded respectively on $\bar{J}$, independently of $\varepsilon$. If any $\lambda_{i}$ has its real part identically zero, let $\vec{J}$ be bounded independently of $\varepsilon$. Then there exist $m$ solutions $\mathbf{x}_{r}, \mathbf{y}_{r}$ of (1.6) and (1.7) that are linearly independent on $\bar{J}$ and which take the form

$$
\begin{aligned}
& \mathbf{x}_{r}=\varepsilon^{-1} X Z \int^{t} Z^{-1} X^{-1} B \mathbf{y}_{r}, \\
& \mathbf{y}_{r}=\chi\left(\mathbf{e}_{r}+o(1)\right),
\end{aligned}
$$

$r=n+1, \ldots, n+m$, where $\mathrm{e}_{r}$, are the usual basis vectors in $R^{m}$, and the $o(1)$ are terms that vanish with $\varepsilon$ uniformly on $\bar{J}$.

Proof. The solutions $y_{r}$ have been constructed as above. The $x_{r}$ are given from (3.1) as

$$
\mathbf{x}_{r}=\varepsilon^{-1} X Z \int_{\mu_{s}}^{t} Z^{-1} X^{-1} B \mathbf{y}_{r}
$$

and application of (3.16) together with (3.2) and (1.2) gives the result (3.15).

\section{Application of the constructed basis}

The properties of the particular set of solutions constructed in Sections 2 and 3 strongly suggest that these two sets together form a complete basis for the solution space of (1.6) and (1.7). All that we need to establish is the linear independence of this (full) set.

Thus, suppose that the solutions were not linearly independent on $\bar{J}$. This implies that there exists a non-trivial pair of constant vectors $c_{1} \in R^{n}$ and $c_{2} \in R^{m}$ (or appropriate complex extensions if $A$ has complex eigenvalues in conjugate complex pairs), such that

$$
\left[\begin{array}{l}
x_{1} \cdots x_{n+m} \\
y_{1} \cdots y_{n+m}
\end{array}\right]\left[\begin{array}{l}
c_{1} \\
c_{2}
\end{array}\right]=0
$$

for all $t \in \bar{J}$, where the first $n$ columns of the square matrix are formed from $\mathbf{x}_{r}$ and $\mathbf{y}_{r}$ give by (2.33) and (2.34), respectively, and the last $m$ columns are formed 
from the corresponding vectors in (3.15) and (3.16). It thus follows from Sections 2 and 3 that (4.1) is equivalent to

$$
\left[\begin{array}{cc}
S M\left(I_{n}+o(1)\right) & N \chi^{-1} \\
P & I_{m}+o(1)
\end{array}\right]\left[\begin{array}{l}
Z \mathbf{c}_{1} \\
\chi c_{2}
\end{array}\right]=0
$$

where

$$
P=Z^{-1} \Psi \int^{t} \Psi^{-1} \operatorname{CSMZ}\left(I_{n}+o(1)\right)
$$

and

$$
N=\varepsilon^{-1} X Z \int^{t} Z^{-1} X^{-1} B \chi\left[I_{m}+o(1)\right],
$$

with the appropriate convention regarding the lower terminals of integration. Explicit expressions for the terms denoted by $o(1)$ are available, at some effort, if required. We note that, under the general hypotheses adopted in the two previous sections, $N$ may be algebraically large for small values of $\varepsilon$ at some values of $t$ but that $F$ is uniformly $O(1)$. It suffices to establish linear independence of the $(n+m)$ solutions to show that the determinant of the matrix for which an approximation is given in (4.2) is non-zero, that is,

$$
\operatorname{det}\left[\begin{array}{cc}
S M\left(I_{n}+o(1)\right) & N \chi^{-1} \\
P & I_{m}+o(1)
\end{array}\right] \neq 0 .
$$

We note that

$$
\operatorname{det}\left[\begin{array}{cc}
S M I_{n} & N_{0} \\
0 & I_{m}
\end{array}\right]
$$

is bounded away from zero for any matrix $N_{0}$ whose elements are bounded independently of $\varepsilon$ and for such a matrix it will be possible to use continuity arguments to establish (4.5) provided one can select a value of $t$ for which $N$ is bounded independently of $\varepsilon$. For then the solutions would be linearly independent throughout the interval. Where $A$ has no purely imaginary eigenvalues, the Laplace method [1] may be applied to $N$. It suffices to choose a value of $t$, not an end-point (indeed, strictly, bounded away from an end-value by an amount independent of $\varepsilon$ ) and not a zero of $\operatorname{Re} \lambda_{i}$, for any $\lambda_{i}$, and then, for such $t$, estimates are readily obtained in the form

$$
P=\varepsilon C Z^{-1} \bar{X} Z+o(\varepsilon)
$$

and

$$
N=-A^{-1} B \chi+o(1),
$$

respectively, when $\bar{X}$ has analogous structure to (1.3). In this case, the linear independence is established. 
When purely imaginary eigenvalues are involved, the situation is somewhat more complex, and a much more careful scrutiny of (4.4) is required. We observe that integrals of the form

$$
\exp (-i \eta / \varepsilon) \int_{t_{0}}^{t} h \exp (i \eta / \varepsilon)
$$

have significant contributions from both end-points, as well as from any points of stationary phase in $\left[t_{0}, t_{1}\right]$. Moreover, unlike the case of the Laplace integral, these contributions persist throughout all $\left[t_{0}, t_{1}\right]$ and thus cannot be avoided by a device like that used to establish (4.7) and (4.8). The asymptotic methods described in Erdélyi [1] may be used to show that these contributions are algebraically small in $\varepsilon$, if $h$ has absolutely integrable derivatives, with such integrals being bounded independently of $\varepsilon$.

The significance of these results for the expressions $N$ and $P$ is that the integrals involving $I_{m}$ and $I_{n}$ alone, and corresponding to purely imaginary eigenvalues $\lambda_{r}$, may be treated by these methods, to obtain appropriate estimates, under appropriately stronger assumptions regarding $A, B$ and $C$. Moreover, because points of stationary phase for $\exp \zeta_{r}$, with $\lambda_{r}$ purely imaginary, lie outside the assumptions by which the results of Sections 2 and 3 have been obtained, such estimates yield a bounded result for the first integral in (4.4).

However, the terms appearing as $o(1)$ in (4.3) and (4.4) cannot be treated by such methods as they stand because their derivatives are, in general, large. Considerably more knowledge is needed about the structure of these terms for, in the context of integrals like (4.9), they may be oscillatory, involving a term of the form $\exp (-i \eta / \varepsilon)$ that cancels the $\exp (i \eta / \varepsilon)$ and renders the analysis inappropriate.

A careful scrutiny of the equations (2.15) shows that

$$
\int_{u}^{t} \exp \left(-\zeta_{j}\right) \frac{d}{d s}\left(\lambda_{j}^{-1} I_{j} M^{-1} S^{-1} B \Psi\right)=O(\varepsilon),
$$

if $\dot{A}, \dot{B}$ and $D$ are differentiable with absolutely integrable derivatives, such integrals being bounded independently of $\varepsilon$. The other two integrals occurring can be shown to be $O(\varepsilon)$ if the corresponding image of $\mathbf{e}_{i}$ is $O(\varepsilon)$. Consideration of the circumstances under which this may be true forces us to accept an exceptional case, which arises when two distinct (imaginary) eigenvalue functions take equal values at some point in the range (and thus induce a point of stationary phase in an integral analogous to (4.9)). Otherwise, the necessary estimates can be obtained, to give $N$ bounded values at $\varepsilon \rightarrow 0$.

Similar considerations apply to the operators described by equation (3.9). We do not propose to investigate this exceptional case further here, since we note that at points where distinct imaginary eigenvalue functions meet, our original 
assumptions were already restrictive, so that such points, in a general treatment, need a discussion more general than our present investigation. Thus we are led to the following result.

THEOREM 3. Let Assumptions 1, 2 and the hypotheses of Theorems 1 and 2 hold on $\bar{J}$ for some $\varepsilon \in\left(0, \varepsilon_{0}\right]$, where $\varepsilon_{0}$ is appropriately small. If there are any purely imaginary eigenvalue functions of $A$, let

(i) no distinct pair of such eigenvalue functions be equal, or equal to any complex eigenvalue function at any point of $\bar{J}$ and

(ii) $\dot{A}, \dot{B}$ and $D$ have absolutely integrable derivatives on $\bar{J}$, with such integrals bounded independently of $\varepsilon$.

Then the solutions described by Theorems 1 and 2 form a complete basis for the solution space of the system (1.6) and (1.7).

Proof. The hypotheses given above are sufficient to make $N$ bounded as $\varepsilon \rightarrow 0$ and allow us to use the continuity argument described earlier to deduce linear independence on $\bar{J}$.

\section{REMARKS.}

1. It is worth noting that the above hypotheses are excessive and in fact it may be that careful examination of the estimates of $N$ and $P$ would reveal that the smallness of $P$ would cancel the unboundedness of $N$ to enable us to deduce that the determinant of (4.1) was non-zero for small $\varepsilon$.

2. It can also be seen that, under the assumptions above, the stiff solution corresponding to the purely imaginary eigenvalue function $\lambda_{r}$ has

$$
\mathbf{x}_{r}=\left\{\mathbf{s}_{r}+O(\varepsilon)\right\} \exp \left(\zeta_{r}\right)=\mathbf{X}_{r} \exp \left(\zeta_{r}\right)
$$

while the corresponding $\mathbf{y}_{r}$ is

$$
\mathbf{y}_{r}=\Psi \int_{t_{0}}^{t} \Psi^{-1} C \mathbf{X}_{r} r \exp \left(\zeta_{r}\right)
$$

Except in an $\varepsilon$-neighbourhood of $t_{0}$, in which $y_{r}$ rises from zero to join smoothly on, the value of the integral can be obtained from

$$
\Psi \int_{t_{0}}^{t} \Psi^{-1} C s_{r} \exp \left(\zeta_{r}\right)
$$

which may be evaluated by asymptotic means, with contributions coming from both $t_{0}$ and $t$. The contribution from $t_{0}$ leads to a term $\varepsilon \Psi$, (constant), which could be subtracted out by using a multiple of the non-stiff solutions constructed in Section 3. The difference between such solutions and $\varepsilon \Psi c$ must be included in the $O(\varepsilon)$ term in $\mathbf{X}_{r}$, which serves to emphasize the fact that the basis we have constructed, while mirroring exponential changes, is not really a clear-cut separation of stiff and non-stiff solutions. 
Similarly, if we examine a stiff solution corresponding to an eigenvalue function having positive real part, we have

$$
\mathbf{x}_{r}=\left\{\mathbf{s}_{r}+o(1)\right\} \exp \left(\zeta_{r}\right)
$$

while the corresponding $\mathbf{y}_{r}$, given by

$$
\mathbf{y}_{r}=\Psi \int_{t_{0}}^{t} \Psi^{-1} C \mathbf{X}_{r} \exp \left(\zeta_{r}\right)
$$

may be expressed in the form

$$
\mathbf{y}_{r}=\left\{-C A^{-1} \mathbf{X}_{r}+o(1)\right\} \exp \left(\zeta_{r}\right)
$$

save near $t=t_{0}$.

We turn now to the inhomogeneous system

$$
\begin{aligned}
\varepsilon \dot{\mathbf{x}} & =A \mathbf{x}+B \mathbf{y}+\mathbf{r}_{1}, \\
\dot{\mathbf{y}} & =C \mathbf{x}+D \mathbf{y}+\mathbf{r}_{2},
\end{aligned}
$$

where $\mathbf{r}_{1}$ and $\mathbf{r}_{2}$ are prescribed absolutely integrable functions. Let $S_{0}$ denote the matrix previously denoted by $S M$. Then the previous results give as a fundamental matrix

$$
\left[\begin{array}{cc}
I_{n}+O(\varepsilon) & -A^{-1} B+O(\varepsilon) \\
\varepsilon C A^{-1}+O\left(\varepsilon^{2}\right) & I_{m}+O(\varepsilon)
\end{array}\right]\left[\begin{array}{cc}
S_{0} Z & 0 \\
0 & \chi
\end{array}\right]
$$

which we shall write as

$$
G\left[\begin{array}{cc}
S_{0} Z & 0 \\
0 & \chi
\end{array}\right]
$$

The method of variation of parameters leads to solutions of the form

$$
G\left[\begin{array}{cc}
S_{0} Z & 0 \\
0 & \chi
\end{array}\right] \int^{t}\left[\begin{array}{cc}
Z^{-1} S_{0}^{-1} & 0 \\
0 & \chi^{-1}
\end{array}\right] G^{-1}\left[\begin{array}{c}
\boldsymbol{\varepsilon}^{-1} \mathbf{r}_{1} \\
\mathbf{r}_{2}
\end{array}\right],
$$

and we note that the terminals may be chosen so that for almost all $t$ the integrals involving $Z$ may be estimated locally. Approximate expresssions for $G$ may then be used to generate approximations for the particular solution so generated.

More instructive however is to note that the calculations may be interpreted in a number of ways. Thus let $\mathbf{y}$ denote the $m$-vector portion of the solution generated in this way and $\mathbf{x}$ the corresponding $n$-vector. Then the process of satisfying (4.15) is equivalent to writing $x$ as $G_{1} S_{0} Z \gamma$ and writing $\varepsilon G_{1} S_{0} Z \dot{\gamma}=B y$ $+r_{1}$ so that

$$
\mathbf{x}=\varepsilon^{-1} G_{1} S_{0} Z\left\{\int^{t} Z^{-1} S_{0}^{-1} G_{1}^{-1}\left(B y+r_{1}\right)+\text { constant }\right\}
$$


and choosing the terminals appropriately. The Laplace estimation then gives, save near end-points,

$$
\mathrm{x}=-A^{-1}\left(B \mathbf{y}+\mathrm{r}_{1}\right)+O(\varepsilon) \text { or } A \mathbf{x}+B \mathbf{y}+\mathbf{r}_{1}=O(\varepsilon) .
$$

A similar argument leads to the fact that $y$ satisfies

$$
\dot{\mathbf{y}}=-C\left\{A^{-1} B \mathbf{y}+\mathbf{r}_{1}\right\}+D \mathbf{y}+\mathbf{r}_{2}+O(\varepsilon) .
$$

Thus we are led to the following result.

THEOREM 4. Let no eigenvalue function of $A$ be purely imaginary on $\bar{J}$. Then, for $t$ bounded, independent of $\varepsilon$, away from all points of $\bar{J}$ where we have vanishing real parts or where two different eigenvalue functions have equal real parts, the solutions of the reduced system

$$
\begin{gathered}
A \mathrm{x}_{0}+B \mathrm{y}_{0}+\mathrm{r}_{1}=0, \\
\dot{\mathrm{y}}_{0}=C \mathrm{x}_{0}+D \mathrm{y}_{0}+\mathrm{r}_{2}
\end{gathered}
$$

differ from those of the full system (4.15) and (4.16) by terms which are uniformb small.

REMARK. Note that we have excluded purely imaginary eigenvalues, since they do not yield localized stiff solutions. In straightforward cases of purely imaginary eigenvalues we expect similar results to apply, but we would draw attention to the fact that the result is vitally dependent on the behaviour, not merely the estimates, of integrals of the form

$$
\varepsilon^{-1} \int \exp \left(\zeta_{i}\right) h r_{1}
$$

where $h$ may involve terms like $\varepsilon^{\mu} \exp \left(-\zeta_{i}\right)$, so that caution is required with the heuristic solutions when purely imaginary eigenvalues occur.

We close this section by considering the implications of attempting to extend our basis across the common point of two intervals $\bar{J}_{1}$ and $\bar{J}_{2}$ on which the basic hypotheses necessary for the constructions of Sections 2 and 3 to proceed hold. Such end-points will be characterized as points at which either $\operatorname{Re} \lambda_{i}=0$ for some $i$ (but $\operatorname{Im} \lambda_{i} \neq 0$ ), or there is a change in the ordering of the real parts of the eigenvalue function $\lambda_{i}$ of $A$.

As we have already demonstrated in Part I, the process by which we may join bases on two abutting intervals is a purely algebraic one, depending only on the construction of a suitable transformation matrix that continuously transforms one set of end-values into another. The continuation properties of differential equations then give the desired extension and the only point at which the fact that $\varepsilon$ is small is needed occurs in the construction of such a matrix. 
We may exploit the process used in Part I by considering the non-stiff solutions of Section 3 as stiff solutions having zero eigenvalues. In this way, the original system (1.6) and (1.7) may be considered as a completely stiff system, and the question of continuing the basis from $\bar{J}_{1}=\left[t_{0}, t_{1}\right]$ into $\bar{J}_{2}=\left[t_{1}, t_{2}\right]$ may be considered by the methods adopted for Section 5 of Part I. The crossing set of that reference may be augmented in the present case by appending those eigenvalue functions whose real parts become zero at $t_{1}$ and which are increasining on $\left[t_{0}, t_{1}\right]$. With this interpretation the results established in Part $I$ extend to partially stiff systems.

\section{When $A$ is singular}

Throughout the previous sections we have found it necessary to assume that $A$ is non-singular in order to make the analysis go. In particular, we have been unable to develop even the stiff partial basis in Section 2 even though the case $A$ singular presented no problems at all for the completely stiff system discussed in Part 1. This is perhaps not surprising in view of the fact that, near a zero of an eigenvalue function $\lambda_{\alpha}$, the corresponding eigen-space $R_{\alpha}$ might be considered as locally describing a non-stiff component of the system rather than a stiff one. If this is an appropriate view, then the failure may not be associated with the methods adopted in this series of papers but may represent an inherent difficulty in partially stiff systems. We therefore choose to examine informally the plausible behaviour of solutions as a means of gaining some understanding of the difficulties which would have to be overcome. We do not attempt to prove our assertions but merely to establish that there are an extreme number of possible variations and that an analytic approach aimed at covering all cases would appear doomed to failure. If this view is accepted, then the practical alternative for such systems is to use sufficiently small numerical integration step sizes locally near singular points of $A$ so that there the system need not be considered to be stiff. We hope here to demonstrate that there is little real alternative for large order systems for which numerical integration techniques are required.

Consider, as a first illustration, a system for which $A$ is singular at the right hand end of an interval which we shall label $(0,1)$ for convenience. Then our results apply on the interval $\left(0, t_{0}\right)$, where $t_{0}$ is bounded away from 1 independent of $\varepsilon$. Let us examine what happens if we attempt to use the method of Section 2 by letting $t_{0}$ tend formally to 1 . The specific eigenvalue function $\lambda_{\alpha}$ which vanishes at 1 can vary in both its multiplicity and the order of its zero, and the issue could be further complicated by a failure of $S$ to have rank $n$, a possibility we have avoided throughout this series of papers. If one examines equation (2.26) one recognizes that the point $t=1$ is a singular point for the 
differential equation fixing the appropriate eigenfunction representation $S M$. In fact, singularities of $A$ in equation (2.26) have a similar effect to a local failure of a non-singular differentiable matrix $S$. If the zero of the eigenvalue fnction $\lambda_{\alpha}$ is simple, then $t=1$ is a regular singular point and the behaviour of $M_{\alpha}$ in the neighbourhood of 1 can exhibit all the variety of behaviour associated with such points. It is possible that $M$ can be smooth and regular but it can also be unbounded or singular. For irregular singular points, associated with a higher order zero of $\lambda_{\alpha}$, even more complex behaviour is possible. We content ourselves with looking at cases where $M$ so defined varies as $(1-t)^{\mu}$ for some $\mu$. If we now examine equation (2.24), we see that, if

$$
S^{-1} B C S=\left(S^{-1} B C S\right)_{\alpha},
$$

there is no difficulty introduced there by the singularity of $A$. More generally, one is faced by considering integrals of the form

$$
\exp \left(\zeta_{j}-\zeta_{i}\right) \int^{l} \exp \left(\zeta_{i}-\zeta_{j}\right)(1-s)^{-\mu} H(1-s)^{-1}(1-s)^{\mu} d s,
$$

where, moreover, if $\mu$ is not small, there will be additional strings of terms as only the dominant terms have been included. We merely note that in general such integrals become logarithmically large as $t \rightarrow 1$ so that approach cannot be expected to work. The calculation shows however that significant effects are beginning to enter as $t \rightarrow 1$ before the method has failed.

We believe however that the nature of the results which will apply can be seen by following the heuristic argument underlying our derivation of the integral equations used. Thus, following the argument leading to equation (1.9), we might argue that a stiff solution might reasonably be expected to be given by

$$
\varepsilon \dot{\mathbf{x}}=A \mathbf{x}+B \Psi \int^{t} \Psi^{-1} C \mathbf{x}
$$

For simplicity, let us assume that the eigenvalue function $\lambda_{\alpha}$ is simple and is positive on $(0,1)$, and that scales are chosen so that $\lambda_{\alpha}(1)=-1$. Then the best which can happen is that changes in the eigenvalue function do not matter so that, if we take $z \mathbf{S}_{i}(1)$ as being the significant component, the awkward solution might behave like

$$
\varepsilon \dot{z}=-(1-t) z+h \int^{t} z
$$

where $h$ is derived from $B C(1)$. In the best of circumstances this might serve as a locally dominant operator for the most interesting stiff solution. Standard heuristic arguments suggest that there will be a range of values of $(1-t)$ of order $\varepsilon^{1 / 2}$ in which a balance is to be expected between all three terms in equation (5.2). 
Thus we introduce a new variable $\tau=\varepsilon^{-1 / 2}(1-t)$ and we have

$$
\frac{d z}{d \tau}=\tau z-h_{0}-h \int_{0}^{\tau} z
$$

Here we have used the fact that the point $t=1$ is a regular point of the original system, if not of the transformed asymptotic system, to claim that the integral to $t=1$ has meaning. A differentiation then yields

$$
\frac{d^{2} z}{d \tau^{2}}=\tau \frac{d z}{d \tau}+(1-h) z
$$

For large values of $\tau$, one solution of this equation behaves essentially as $\exp \left(-\tau^{2} / 2\right)$, which is the appropriate representation of $\exp \left(\varepsilon^{-1} \int_{0}^{t} \lambda_{\alpha}\right)$ scaled to its value at $t=1$. The behaviour of the solution near $\tau=0$ is dependent on the value of $h$ and an indication can be seen if the magnitude of $h$ is moderately large. Thus, if $h$ is positive and reasonably large, the solution can be expected to oscillate for moderate values of $\tau$ while, if it is negative and large, the solution is likely to be monotone for moderate values of $\tau$.

If both $m$ and $n$ were 1 we would expect that we could make the argument rigorous but, as the size of the system increased, we must expect that much more complex behaviour is likely to occur. It is for these reasons that we believe that an attempt to develop a general asymptotic theory by analytic means is inappropriate. It is possible that generating integral equations along the lines of (1.9) and (2.5)may prove an effective means of developing local means of numerical integration near singular points of $A$. We would further suggest that a similar approach using fixed bases may also be the appropriate technique for dealing with the neighbourhoods of points where there is local failure of the eigenvectors to span $R^{n}$.

\section{Acknowledgement}

This research was supported in part by the Australian Research Grants Committee.

\section{References}

[1] A. E. Erdélyi, Asymptotic expansions (Dover, New York, 1956).

[2] J. J. Mahony and J. J. Shepherd, "Stiff systems of ordinary differential equations. I. Completely stiff homogeneous equations", J. Austral. Math. Soc. B 23 (1981), 17-51. 
[3] J. J. Mahony and J. J. Shepherd, "Stiff systems of ordinary differential equations. II. Boundary value problems for completely stiff systems", J. Austral. Math. Soc. B 23 (1981), 136-172.

Department of Mathematics

University of Western Australia

Nedlands

Western Australia 6009 